\title{
Strains of the Lactobacillus casei group show diverse abilities for the production of flavor compounds in 2 model systems
}

\author{
Ewelina Stefanovic, ${ }^{*} \dagger$ Anne Thierry, $\ddagger$ Marie-Bernadette Maillard,‡ Andrea Bertuzzi, ${ }^{\star} \S$ Mary C. Rea, ${ }^{*}$ \\ Gerald Fitzgerald,† Olivia McAuliffe, ${ }^{*}$ and Kieran N. Kilcawley*1 \\ *Department of Food Biosciences, Teagasc Food Research Centre, Moorepark, Fermoy, Co. Cork, Ireland P61 C966 \\ †School of Microbiology, University College Cork, Co. Cork, Ireland T12 YT20 \\ fUMR1253 Science et Technologie du Lait et de l'CEuf, INRA, Agrocampus Ouest, 65 rue de Saint Brieuc, 35000 Rennes, France \\ $\S$ School of Food and Nutritional Science, University College Cork, Co. Cork, Ireland T12 Y337
}

\begin{abstract}
Cheese flavor development is directly connected to the metabolic activity of microorganisms used during its manufacture, and the selection of metabolically diverse strains represents a potential tool for the production of cheese with novel and distinct flavor characteristics. Strains of Lactobacillus have been proven to promote the development of important cheese flavor compounds. As cheese production and ripening are long-lasting and expensive, model systems have been developed with the purpose of rapidly screening lactic acid bacteria for their flavor potential. The biodiversity of 10 strains of the Lactobacillus casei group was evaluated in 2 model systems and their volatile profiles were determined by gas chromatography-mass spectrometry. In model system 1 , which represented a mixture of free AA, inoculated cells did not grow. In total, 66 compounds considered as flavor contributors were successfully identified, most of which were aldehydes, acids, and alcohols produced via AA metabolism by selected strains. Three strains (DPC2071, DPC3990, and DPC4206) had the most diverse metabolic capacities in model system 1 . In model system 2, which was based on processed cheese curd, inoculated cells increased in numbers over incubation time. A total of 47 compounds were identified, and they originated not only from proteolysis, but also from glycolytic and lipolytic processes. Tested strains produced ketones, acids, and esters. Although strains produced different abundances of volatiles, diversity was less evident in model system 2, and only one strain (DPC4206) was distinguished from the others. Strains identified as the most dissimilar in both of the model systems could be more useful for cheese flavor diversification.
\end{abstract}

Key words: Lactobacillus, flavor, biodiversity, model system

Received December 6, 2016.

Accepted April 22, 2017.

${ }^{1}$ Corresponding author: kieran.kilcawley@teagasc.ie

\section{INTRODUCTION}

Formation of cheese flavor is a complex process, which results mainly from the metabolic activities of microorganisms present during cheese manufacture (Marilley and Casey, 2004; Smit et al., 2005). Lactic acid bacteria are the most commonly found bacteria in dairy products and their metabolic diversity represents a potential tool for flavor diversification and improvement (Smit et al., 2005). Nonstarter lactic acid bacteria (NSLAB) that originate from the cheese-making environment dominate the cheese microbiota during ripening (Vaughan et al., 2001). The metabolic activity of NSLAB during ripening leads to the production of compounds contributing to the flavor characteristics of cheese (Fitzsimons et al., 2001; Banks and Williams, 2004), and this effect has been shown to be highly strain specific (Randazzo et al., 2007; Bouton et al., 2009; Pogačić et al., 2016).

The mesophilic lactobacilli dominate the NSLAB flora of cheese, as seen in a broad survey of NSLAB diversity, where 18 species of mesophilic lactobacilli were detected in 38 cheese varieties with Lactobacillus paracasei and Lactobacillus plantarum as the most prevalent species. These are considered as very adaptable to the cheese environment, and along with Lactobacillus casei, Lactobacillus curvatus, and Lactobacillus rhamnosus, represent the core species of the nonstarter microbiota (Gobbetti et al., 2015). Adjunct cultures are essentially selected strains of NSLAB that are added to cheese milk with the purpose of controlling the indigenous NSLAB population, and thus, directing the development of desired cheese flavor compounds (Milesi et al., 2010; Singh and Singh, 2014). Strains of the Lactobacillus casei group (L. casei, L. paracasei, and L. rhamnosus) have been successfully used as adjuncts, solely or in combination with other lactobacilli in Cheddar cheese manufacture (Crow et al., 2001; Swearingen et al., 2001; Coolbear et al., 2008).

Ideally, the evaluation of the flavor-forming ability of new strains should be performed in cheese-making 
trials, but this is only practical as a final step as such trials are expensive, laborious, and time consuming (Milesi et al., 2007). To a certain extent, model systems mimic some aspects of the cheese ripening environment and enable rapid assessment of the development of the cheese microbiota and the resultant biochemical processes. Several types of cheese models have been developed based on miniature cheese production (Di Cagno et al., 2006; Milesi et al., 2008; Cavanagh et al., 2014), cheese slurry (Smit et al., 1995), or processed curd (Pogačić et al., 2015; Velez et al., 2015). In addition, synthetic systems that consist of solutions of a similar content to cheese could be used as model systems, such as those based on AA-rich media (Engels and Visser, 1996; Kieronczyk et al., 2001; van de Bunt et al., 2014). Besides these, cheese serum extracts (Peralta et al., 2014), freeze-drying of cheese and extraction with water (Budinich et al., 2011), or lysate of cells (Sgarbi et al., 2013) were also successfully used as cheese models. A model based on miniature cheeses made from as little as $1.7 \mathrm{~mL}$ of milk enabled screening of flavor-forming capacities of microorganisms (Bachmann et al., 2009). In most cheese or curd-based model systems, inoculated cells increased in numbers, whereas in synthetic medium model systems, inoculated cells were not growing (Kieronczyk et al., 2001; van de Bunt et al., 2014). Additionally, cell-free extracts have been used as a source of enzymes to investigate the flavor-forming capacity of Lactococcus lactis (Engels and Visser, 1996).

The aim of this study was to evaluate the diversity between strains of the $L$. casei group based on determination of their volatile profiles generated in 2 model systems: a model consisting of a mixture of free AA and a processed curd model. Afterward, the strain diversity was mapped using a chemometric approach, which showed different abilities of strains for volatile production in the 2 model systems used.

\section{MATERIALS AND METHODS}

\section{Bacterial Strains}

Ten strains of the Lactobacillus casei group of dairy origin were used in this study (DPC1116, DPC2068, DPC2071, DPC3990, DPC4026, DPC4206, DPC4536, DPC5408, DPC6753, and DPC6800). Strains used in this study were previously confirmed (by $16 \mathrm{~S}$ rRNA PCR) to belong to species L. casei or L. paracasei and were selected based on genomic profiles (pulsed-field gel electrophoresis) and biochemical characterization (activities of proteolytic cascade enzymes) of a set of 310 isolates obtained from the DPC Culture Collection held at the Teagasc Food Research Centre, Moorepark, Cork, Ireland (Stefanovic et al., 2017). Strains were kept frozen at $-80^{\circ} \mathrm{C}$ in de Man, Rogosa, and Sharpe broth (MRS, Oxoid, UK) with $20 \%$ (vol/vol) of glycerol, and before the experiment they were grown on MRS agar plates at $30^{\circ} \mathrm{C}$ in aerobic conditions.

\section{Model System 1: Resting Cells in Medium Containing Free Amino Acids}

Model system 1 (MS1) consisted of a suspension of nongrowing cells in a concentrated (35\% (wt/vol) AA-rich medium Bacto Tryptone [BD, Franklin Lakes, NJ; containing a minimal level of total carbohydrates $(4.3 \mathrm{mg} / \mathrm{g})]$ supplemented with $12 \mathrm{~g} / \mathrm{L}$ of $\mathrm{NaCl}$. This medium was chosen based on the composition of a similar model described by van de Bunt et al. (2014). Medium for MS1 was prepared from the same batch of Bacto Tryptone, and after addition of $\mathrm{NaCl}$, it was autoclaved $\left(121^{\circ} \mathrm{C}, 15 \mathrm{~min}\right)$. Cell manipulation was performed as described by van de Bunt et al. (2014), with some modifications. Briefly, strains were pre-incubated for $18 \mathrm{~h}$ at $30^{\circ} \mathrm{C}$ in MRS broth, re-inoculated ( $1 \% \mathrm{vol} /$ vol) in $500 \mathrm{~mL}$ of MRS broth, and incubated for 24 $\mathrm{h}$ at $30^{\circ} \mathrm{C}$. Cells were centrifuged $(4,000 \times g, 10 \mathrm{~min}$, $4^{\circ} \mathrm{C}$ ), washed twice with $0.1 \mathrm{~mol} / \mathrm{L}$ phosphate buffer $\mathrm{pH} 6$, and finally resuspended in $5 \mathrm{~mL}$ of the same buffer containing $15 \%$ glycerol and kept at $-80^{\circ} \mathrm{C}$ until required. Thawed cell suspensions $(1 \mathrm{~mL})$ were added in $9 \mathrm{~mL}$ of the prepared AA-rich medium including $10 \mu \mathrm{L}$ of a vitamin and microelement solution, which contained $2 \mathrm{mg}$ of biotin, $4.8 \mathrm{mg}$ of Ca-pantothenate, 8 $\mathrm{mg}$ of thiamine, $8 \mathrm{mg}$ of $\mathrm{FeSO}_{4}, 1.6 \mathrm{mg}$ of $\mathrm{MgSO}_{4}$, and 8 $\mathrm{mg}$ of $\mathrm{MnSO}_{4}$ dissolved in $4 \mathrm{~mL}$ of deionized water and filter sterilized (Filtropur S syringe filter, $0.45 \mu \mathrm{m}$ pore size, Sarstedt, Wexford, Ireland). Inoculated samples were incubated for $24 \mathrm{~h}$ at $30^{\circ} \mathrm{C}$. For cell enumeration, samples of $100 \mu \mathrm{L}$ were taken before and after incubation of the inoculated model system (at time $=0 \mathrm{~h}$ and time $=24 \mathrm{~h}$ ) and serially diluted before plating on MRS agar followed by incubation at $30^{\circ} \mathrm{C}$ for $72 \mathrm{~h}$. After incubation, $\mathrm{pH}$ values of the samples were also measured. Samples were kept at $-80^{\circ} \mathrm{C}$ until volatile analysis was performed. The control consisted of an uninoculated model system. Both the test strains and the uninoculated control were evaluated in triplicate.

\section{Model System 2: Growing Cells in Processed Curd}

Model system 2 (MS2) was prepared as previously described (Pogačić et al., 2015) with the following modifications, to achieve final concentrations of $1 \mathrm{~g} / \mathrm{L}$ of lactose and $5.3 \%$ (wt/wt) salt in moisture. A solution containing $1.48 \mathrm{~g} / \mathrm{L}$ of peptone and $1.48 \mathrm{~g} / \mathrm{L}$ of lactose and a solution of $254.25 \mathrm{~g} / \mathrm{L}$ of $\mathrm{NaCl}$ were prepared in advance and autoclaved $\left(121^{\circ} \mathrm{C}, 15 \mathrm{~min}\right)$. The Ched- 
dar cheese curd $[\mathrm{pH} 5.31, \mathrm{NaCl} 2.45 \%$ (wt/wt), water activity $\left(\mathrm{a}_{\mathrm{w}}\right)$ 0.948, moisture $38.1 \%$ (wt/wt), fat $31.2 \%$ (wt/wt)] and the peptone-lactose solution were mixed in a 1:2 ratio (wt/wt) and blended in a Waring blender (Waring, Stamford, CT), over 4 cycles for $30 \mathrm{~s}$ at low speed and 2 cycles for $30 \mathrm{~s}$ at high speed. In each tube, $10 \mathrm{~g}$ of the curd mixture was weighed and tubes were autoclaved $\left(110^{\circ} \mathrm{C}, 15 \mathrm{~min}\right)$. Subsequently, $1.34 \mathrm{~mL}$ of the sterile $\mathrm{NaCl}$ solution was added to each tube to prevent formation of curd clogs. Strains were grown at $30^{\circ} \mathrm{C}$ for $24 \mathrm{~h}$ in MRS broth. Cultures were diluted to $\mathrm{OD}_{650 \mathrm{~nm}}$ of 0.35 to 0.45 (approximately $10^{7}-10^{8} \mathrm{cfu} /$ $\mathrm{mL}$ ), and the dilutions were used for inoculation of pre-cultures in the model system at $1 \%$ (vol/vol). Preculture tubes (triplicate for each strain) were incubated for $24 \mathrm{~h}$ at $30^{\circ} \mathrm{C}$ after which enumeration of pre-cultures was achieved by serial dilutions and plate counting on MRS agar at $30^{\circ} \mathrm{C}$ for $72 \mathrm{~h}$. Fresh tubes with curd were inoculated at $1 \%$ (vol/vol) of pre-culture and incubated for $14 \mathrm{~d}$ at $30^{\circ} \mathrm{C}$, after which $\mathrm{pH}$ and cell counts were determined. Samples were kept at $-80^{\circ} \mathrm{C}$ until volatile analysis was performed. The control consisted of an uninoculated model system. As above, both the strains and the control were evaluated in triplicate.

\section{Gas Chromatography-Mass Spectrometry Analysis of Volatiles}

A 2.5-mL sample of the corresponding model system (triplicate per strain per model system) was placed in a 20-mL Perkin Elmer sealed vial (Perkin Elmer, Courtaboeuf, France). Headspace trap GC-MS analysis was performed using a Clarus 680 GC coupled with Clarus $600 \mathrm{~T}$ quadrupole MS (Perkin Elmer) as previously described (Pogačić et al., 2015), with modifications. Samples were warmed for $15 \mathrm{~min}$ to $65^{\circ} \mathrm{C}$ and volatiles were extracted at $207 \mathrm{kPa}$ pressure maintained in the vial for 1 min with the carrier gas (helium), before being adsorbed on a Tenax trap (Perkin Elmer) at $35^{\circ} \mathrm{C}$. The trap load was performed twice for each vial. The trap was heated at $250^{\circ} \mathrm{C}$ for 0.1 min and backflushed with helium at $89 \mathrm{kPa}$, leading to desorption of the volatiles. Volatiles were then separated on a Stabilwax MS capillary column $(30 \mathrm{~m} \times 0.25 \mathrm{~mm} \times 0.25 \mu \mathrm{m}$; Restek, Bellefonte, PA), with helium as the mobile phase. The temperature of the oven was initially $35^{\circ} \mathrm{C}$, maintained for $10 \mathrm{~min}$, then increased at $5^{\circ} \mathrm{C} / \mathrm{min}$ up to $230^{\circ} \mathrm{C}$. The MS was operated in the scan mode (scan time $0.2 \mathrm{~s}$, interscan delay $0.1 \mathrm{~s}$ ) within a mass range of $\mathrm{m} / \mathrm{z} 50$ to 300. Ionization was performed by electronic impact at $70 \mathrm{eV}$. The samples were injected in a random order, with standards and blank samples (boiled deionized water) to monitor possible carryover and MS drift, as previously described (Pogačić et al., 2015).

\section{Chemometric Data Processing and Identification of Compounds}

Chromatographic data were processed by the XCMS package of $\mathrm{R}$ statistical software (Smith et al., 2006) to convert GC-MS raw data to time- and mass-aligned data, providing, for each sample, the abundances for several signals (pair of mass fragment and retention time). Analysis of volatiles was semiquantitative, and results were based on abundance (peak area) only. The mean coefficient of variation of the analysis of volatile, calculated based on analysis of standards injected during GC runs, was approximately $17 \%$. Volatiles were identified by comparison of mass spectra and linear retention indices with those of authentic standards, or tentatively identified on the basis of mass spectral data using the NIST 2008 Mass Spectral Library (Scientific Instrument Services, Ringoes, NJ). Where possible, in both data sets signals of same mass were used for the same compounds; in other cases, signals with the highest abundance were chosen. Some of the signals present in XCMS data sets could not be related to any compound or the percentages of identifications were considered unsatisfactory (approximately $<50 \%$ ). The compounds of interest were selected according to previously published review of compounds considered as main flavor contributors in cheese (Curioni and Bosset, 2002).

\section{Statistical Analysis}

Differences in cell counts $\left(\log _{10}\right)$ and $\mathrm{pH}$ values before and after incubation were tested by ANOVA performed using statistical software R (https://www.r-project. org/). The ANOVA was also performed on selected signals to determine the presence of significant differences between the cultures. Means were compared using the least significant difference test. Compounds with significant differences in abundances in cultures including the control, were further evaluated. Principal component analysis (PCA) was performed on selected compounds after Pareto scaling using package FactomineR of the $R$ software.

\section{RESULTS}

\section{Resting Cells in Amino Acid-Rich Medium Show Diverse Metabolic Activities}

The cell enumerations in MS1 did not significantly vary during incubation, except for DPC2071, which showed a $0.43 \log _{10}$ unit decrease (Table 1). The $\mathrm{pH}$ values after incubation did not significantly differ from the $\mathrm{pH}$ values of the control, except for 2 cultures 
Table 1. Cell enumeration in 2 model systems before and after incubation ${ }^{1}$

\begin{tabular}{lccccc}
\hline & \multicolumn{2}{c}{ Model system 1} & & \multicolumn{2}{c}{ Model system 2} \\
\cline { 2 - 3 } \cline { 5 - 6 } Strain & $\mathrm{t}=0 \mathrm{~h}$ & $\mathrm{t}=24 \mathrm{~h}$ & & $\mathrm{t}=0 \mathrm{~h}$ & $\mathrm{t}=14 \mathrm{~d}$ \\
\hline DPC1116 & $9.96 \pm 0.21$ & $9.875 \pm 0.09$ & & $\mathbf{6 . 7 1} \pm \mathbf{0 . 0 5}$ & $\mathbf{8 . 3 5} \pm \mathbf{0 . 1 6}$ \\
DPC2068 & $9.49 \pm 0.06$ & $9.62 \pm 0.08$ & & $\mathbf{6 . 7 1} \pm \mathbf{0 . 0 5}$ & $\mathbf{7 . 7 9} \pm \mathbf{0 . 1 7}$ \\
DPC2071 & $\mathbf{1 0 . 2 2} \pm \mathbf{0 . 2 1}$ & $\mathbf{9 . 7 9} \pm \mathbf{0 . 0 9}$ & & $\mathbf{6 . 6 7} \pm \mathbf{0 . 0 7}$ & $\mathbf{8 . 1 3} \pm \mathbf{0 . 1 8}$ \\
DPC3990 & $10.05 \pm 0.11$ & $10.02 \pm 0.07$ & & $\mathbf{6 . 6 4} \pm \mathbf{0 . 0 6}$ & $\mathbf{8 . 1 0} \pm \mathbf{0 . 1 4}$ \\
DPC4026 & $9.84 \pm 0.04$ & $9.68 \pm 0.17$ & & $\mathbf{6 . 6 5} \pm \mathbf{0 . 1 8}$ & $\mathbf{7 . 9 2} \pm \mathbf{0 . 0 5}$ \\
DPC4206 & $10.02 \pm 0.04$ & $9.95 \pm 0.06$ & & $\mathbf{6 . 6 7} \pm \mathbf{0 . 0 9}$ & $\mathbf{7 . 6 3} \pm \mathbf{0 . 3 3}$ \\
DPC4536 & $9.97 \pm 0.07$ & $9.90 \pm 0.10$ & & $\mathbf{6 . 3 5} \pm \mathbf{0 . 1 9}$ & $\mathbf{7 . 4 7} \pm \mathbf{0 . 4 1}$ \\
DPC5408 & $9.80 \pm 0.08$ & $9.79 \pm 0.13$ & & $\mathbf{5 . 8 7} \pm \mathbf{0 . 7 3}$ & $\mathbf{7 . 9 2} \pm \mathbf{0 . 2 2}$ \\
DPC6753 & $9.99 \pm 0.13$ & $9.85 \pm 0.20$ & & $\mathbf{6 . 2 0} \pm \mathbf{0 . 5 5}$ & $\mathbf{7 . 6 9} \pm \mathbf{0 . 1 3}$ \\
DPC6800 & $10.1 \pm 0.04$ & $10.06 \pm 0.10$ & & $\mathbf{6 . 7 5} \pm \mathbf{0 . 0 5}$ & $\mathbf{7 . 3 3} \pm \mathbf{0 . 1 7}$ \\
\hline
\end{tabular}

${ }^{1}$ All strains belong to Lactobacillus casei group. Results are presented as mean values \pm SD of triplicate experiments. Strains that showed a significant difference in cell numbers after incubation according to LSD test are presented in bold $(P<0.05) . \mathrm{t}=$ time.

(DPC2071 and DPC3990) that showed a slight decrease $(<0.2 \mathrm{pH}$ units; Table 2$)$.

Analysis of chromatograms revealed 66 potential flavor-contributing compounds (Table 3). According to the statistical analysis (ANOVA and LSD test), 30 compounds were present in statistically different abundances in cultures, including the control $(P<0.05)$. The ratio of the highest and the lowest values of abundance for a single compound between the cultures (ratio B, Table 3) ranged between 1.5 for butyl decanoate and 111.5 for 2-ethyl-2-hexenal. It was apparent that volatile compounds were present in higher abundances in cultures than in the control, except dimethyl trisulfide (DMTS), dimethyl tetrasulfide, and nonanal. Additionally, chromatograms of all cultures and the control showed a stretched peak of butan-1-ol and confirmed that this alcohol was present in high abundance in all MS1 samples. Several strains showed robust metabolic characteristics when incubated in MS1 (Supplemental

Table 2. $\mathrm{pH}$ values of cultures and control measured at the end of incubation of strains of Lactobacillus casei group in the 2 models $^{1}$

\begin{tabular}{lll}
\hline Strain & Model system 1 & Model system 2 \\
\hline DPC1116 & $6.95 \pm 0.02^{\mathrm{ab}}$ & $5.12 \pm 0.03^{\mathrm{b}}$ \\
DPC2068 & $6.95 \pm 0.03^{\mathrm{ab}}$ & $5.01 \pm 0.04^{\mathrm{f}}$ \\
DPC2071 & $6.82 \pm 0.11^{\mathrm{c}}$ & $5.07 \pm 0.01^{\mathrm{bcde}}$ \\
DPC3990 & $6.87 \pm 0.04^{\mathrm{bc}}$ & $5.01 \pm 0.02^{\mathrm{ef}}$ \\
DPC4026 & $7.05 \pm 0.03^{\mathrm{a}}$ & $5.08 \pm 0.02^{\mathrm{bcd}}$ \\
DPC4206 & $6.93 \pm 0.08^{\mathrm{abc}}$ & $5.05 \pm 0.03^{\mathrm{cdef}}$ \\
DPC4536 & $6.96 \pm 0.14^{\mathrm{ab}}$ & $5.09 \pm 0.08^{\mathrm{bc}}$ \\
DPC5408 & $6.93 \pm 0.13^{\mathrm{abc}}$ & $5.03 \pm 0.01^{\mathrm{def}}$ \\
DPC6753 & $7.01 \pm 0.04^{\mathrm{a}}$ & $5.06 \pm 0.02^{\mathrm{cde}}$ \\
DPC6800 & $7.01 \pm 0.04^{\mathrm{a}}$ & $5.05 \pm 0.00^{\mathrm{cdef}}$ \\
Control & $7.01 \pm 0.01^{\mathrm{a}}$ & $5.28 \pm 0.02^{\mathrm{a}}$ \\
\hline
\end{tabular}

${ }^{\mathrm{a}-\mathrm{f}}$ Results of $\mathrm{pH}$ values sharing the same letter in the column corresponding to model system 1 or model system 2 do not significantly differ according to the LSD test $(P<0.05)$.

${ }^{1}$ Results are presented as mean values $\pm \mathrm{SD}$ of triplicate experiments. The control consisted of uninoculated model systems.
Table S1; https://doi.org/10.3168/jds.2016-12408). Strain DPC4206 produced 8 compounds in the highest relative abundances (butanal, S-methyl-thioacetate, butyl butanoate, 2-ethyl-2-hexenal, butanoic acid, 3-methyl-butanoic acid, undecan-2-ol, hexanoic acid), 7 of which were significantly higher compared with abundances in all other cultures, including the control (except for hexanoic acid). Strain DPC2071 produced 3-methyl-butan-1-ol, 3-hydroxy-butan-2-one (acetoin), acetic acid, octan-1-ol, and butyl decanoate in the highest abundances, and the abundance of 3-hydroxybutan-2-one was significantly higher than in other cultures, including the control. Strain DPC3990 produced 6 compounds in the highest abundances (hexan-1-ol, 3-methyl-hexan-1-ol, butanedioic acid dimethyl ester, nonan-2-one, undecan-2-one, tridecan-2-one, and benzeneacetic acid butyl ester, all of which except nonan2-one and benzeneacetic acid butyl ester were produced in significantly higher abundances compared with the production by all other strains, including the control (Supplemental Table S1; https://doi.org/10.3168/ jds.2016-12408).

In the PCA plot generated for MS1 using the abundance of 30 volatiles across all cultures and the control (Figure 1), the first 2 axes accounted for $73.5 \%$ of the total variability. Dimension 1 (PC1), describing $60.3 \%$ variability was related to the abundance of the majority of flavor compounds. The variables factor map shows 14 variables that were the best represented in dimensions 1 and 2 . The variables were positively associated with PC1, except for DMTS, dimethyl tetrasulfide, and nonanal, which were negatively associated with $\mathrm{PC} 1$. Dimension 2 (PC2), describing $13.2 \%$ variability, was positively related to butanal, butanoic acid, butyl butanoate, 2-ethyl-2-hexenal, and dimethyl tetrasulfide, whereas other variables showed poor correlation with PC2. The control appeared in the left quadrant and 
Table 3. Compounds identified in model system 1 along with linear retention indices (LRI) used for compound identification ${ }^{1}$

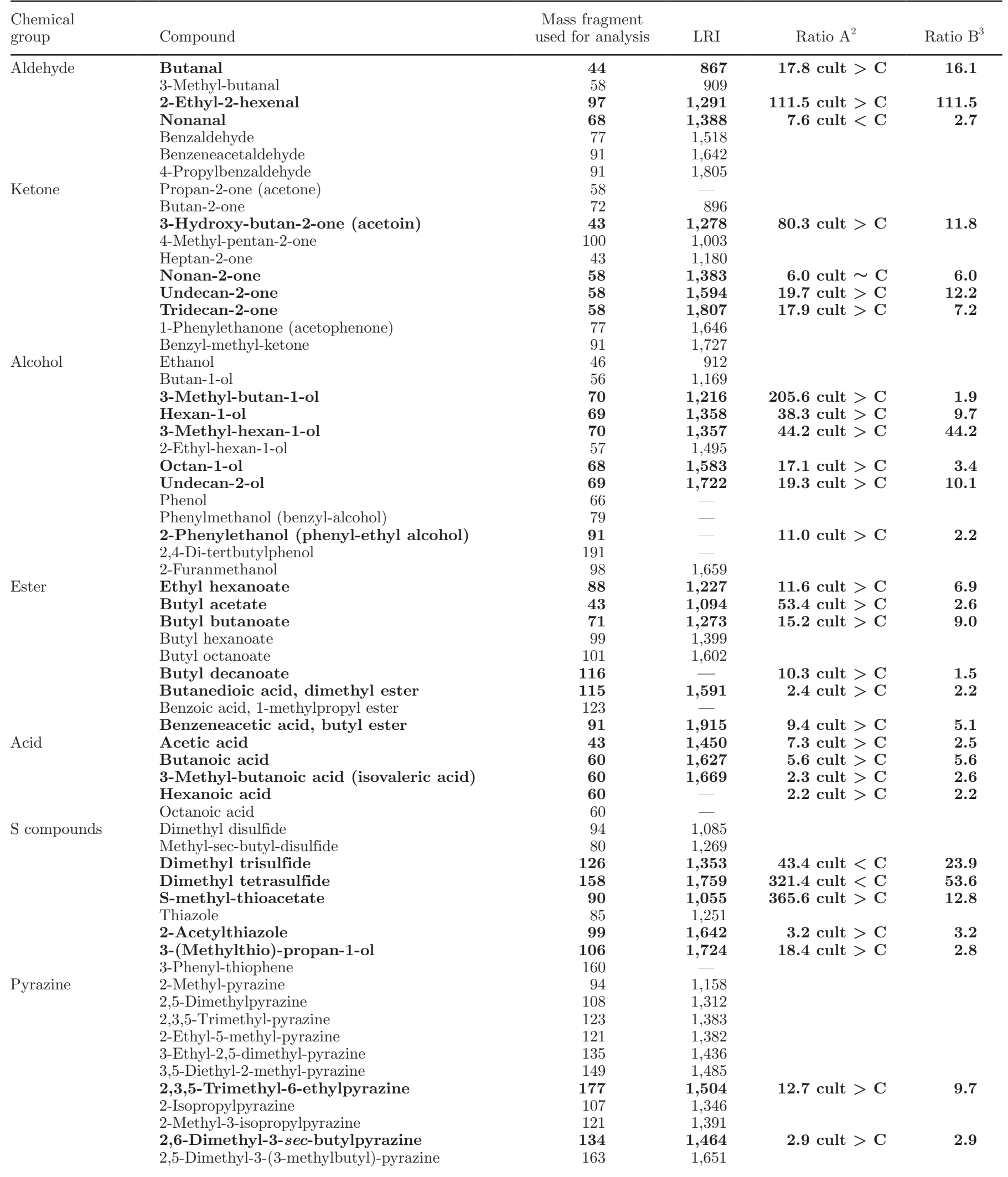


Table 3 (Continued). Compounds identified in model system 1 along with linear retention indices (LRI) used for compound identification ${ }^{1}$

\begin{tabular}{|c|c|c|c|c|c|}
\hline $\begin{array}{l}\text { Chemical } \\
\text { group }\end{array}$ & Compound & $\begin{array}{l}\text { Mass fragment } \\
\text { used for analysis }\end{array}$ & LRI & Ratio $A^{2}$ & Ratio $\mathrm{B}^{3}$ \\
\hline $\mathrm{N}$ compound & $\begin{array}{l}\text { Benzonitrile } \\
\text { Indole }\end{array}$ & $\begin{array}{l}103 \\
117\end{array}$ & 1,601 & & \\
\hline
\end{tabular}

${ }^{1}$ The control was an uninoculated model under the same conditions. Cultures and control were tested in triplicate. Compounds that exhibited significant differences in abundances in cultures, including the control $(P<0.05)$, are presented in bold.

${ }^{2}$ Ratio A presents the maximal ratio of abundance of a compound, between the cultures and the control: (abundance in cultures)/(abundance in control), if cult $>\mathrm{C}$; or (abundance in control)/(abundance in cultures), if cult $<\mathrm{C}$; or cult $\sim \mathrm{C}$, if abundance of compound in the control was higher than in some cultures, but lower than in others.

${ }^{3}$ Ratio B presents the maximal ratio of abundance of a compound between the cultures.

was negatively associated with most variables. The PC1 was positively related to most of the strains, except for strains DPC6753 and DPC4026, and PC2 was positively related to DPC4206 and DPC4536, and negatively to DPC6800 and DPC2071.

Based on the PCA plot, 3 strains were distinguished from others. The DPC4206 strain was positioned in the upper right quadrant and was associated with the production of butanal, butanoic acid, butyl butanoate, and 2-ethyl-2-hexenal, whereas DPC2071 appeared to be associated with the highest production of acetic acid, butyl decanoate, 3-hydroxy-butan-2-one, octan-1-ol, and 3-methyl-butan-1-ol, in the lower right quadrant. The DPC3990 strain was equally separated as the 2 aforementioned strains in PC1 and was associated with the highest abundances of nonan-2-one, hexan-1-ol, undecan-2-one, tridecan-2-one, and benzeneacetic acid butyl ester.

\section{Growing Cells in Processed Curd Show Limited Diversity in Volatiles Production}

In MS2, cell numbers significantly increased in all cultures (Table 1), and the mean increase was $1.3 \log _{10}$ units, whereas measured $\mathrm{pH}$ values after incubation showed a significant decrease for all cultures compared with the $\mathrm{pH}$ value of the control, with mean of decrease of 0.22 (Table 2).

The volatile profiles of cultures revealed a total of 47 potential flavor compounds and the abundances of 10 of these showed significant differences between the cultures, including the control $(P<0.05$; Table 4$)$. The (a) Individuals factor map (PCA)

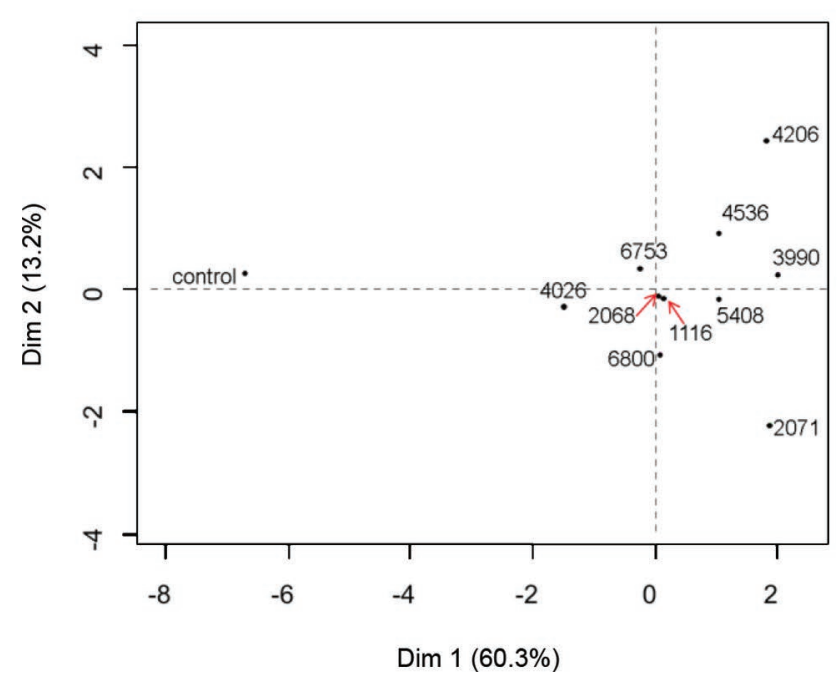

(b) Variables factor map (PCA)

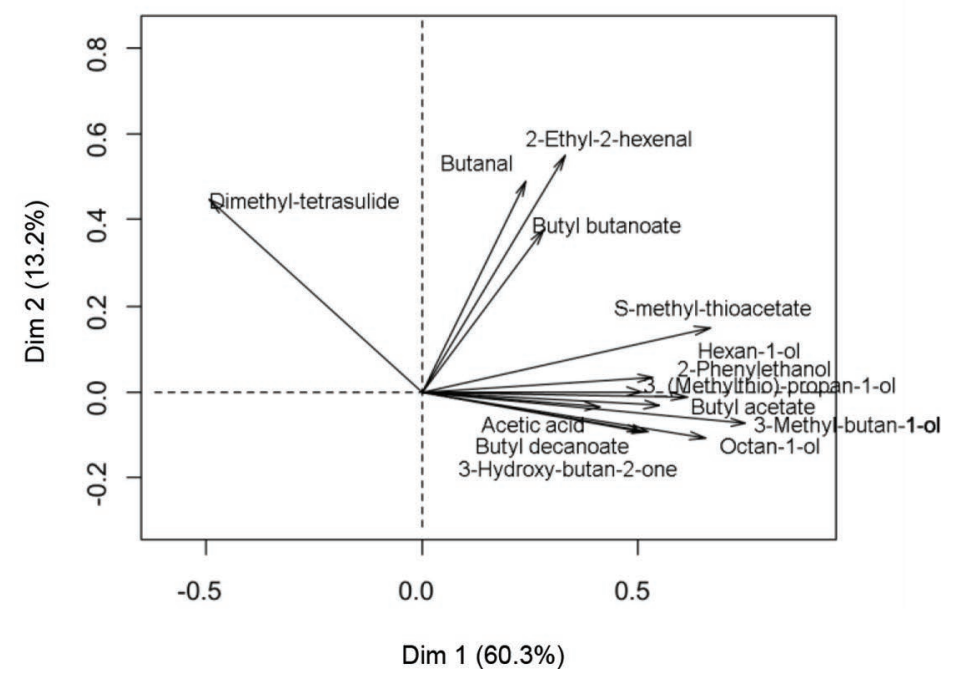

Figure 1. Individual factor map (a) and variable factor map (b) of principal component analysis (PCA) on 30 volatile compounds produced by 10 strains of Lactobacillus casei group in model system 1 incubated for $24 \mathrm{~h}$ at $30^{\circ} \mathrm{C}$. The control was an uninoculated model system incubated under the same conditions. Cultures and control were tested in triplicate. The variables poorly represented in this plot (square cosinus limt below 0.8 ) are not shown, and only the 14 variables that are the best represented in dimension 1 (Dim 1) and dimension 2 (Dim 2) are shown. The DPC prefix has been removed from the strain name to avoid potential illegibility of the figure. Color version available online. 
ratio of the highest and the lowest values of abundance between the cultures for a single compound (ratio B, Table 4) ranged between 1.3 for 2-phenylethanol to 3.2 for 2,3-butanedione (diacetyl). Of the 10 compounds for which significant differences in signal abundances were observed, aldehydes were present in lower abundances in cultures than in the control, whereas com- pounds present in higher abundances in the cultures included acetic acid, 2-phenylethanol, ethyl acetate, 2,3-butanedione, and 1-hydroxy-propane-2-one. Although mean comparison showed that in the case of 2-methyl-propanal, hexanal, and 2-phenylethanol, there were significant differences in the relative abundances between the cultures and the control, no sig-

Table 4. Compounds identified in model system 2 along with linear retention indices (LRI) used for compound identification ${ }^{1}$

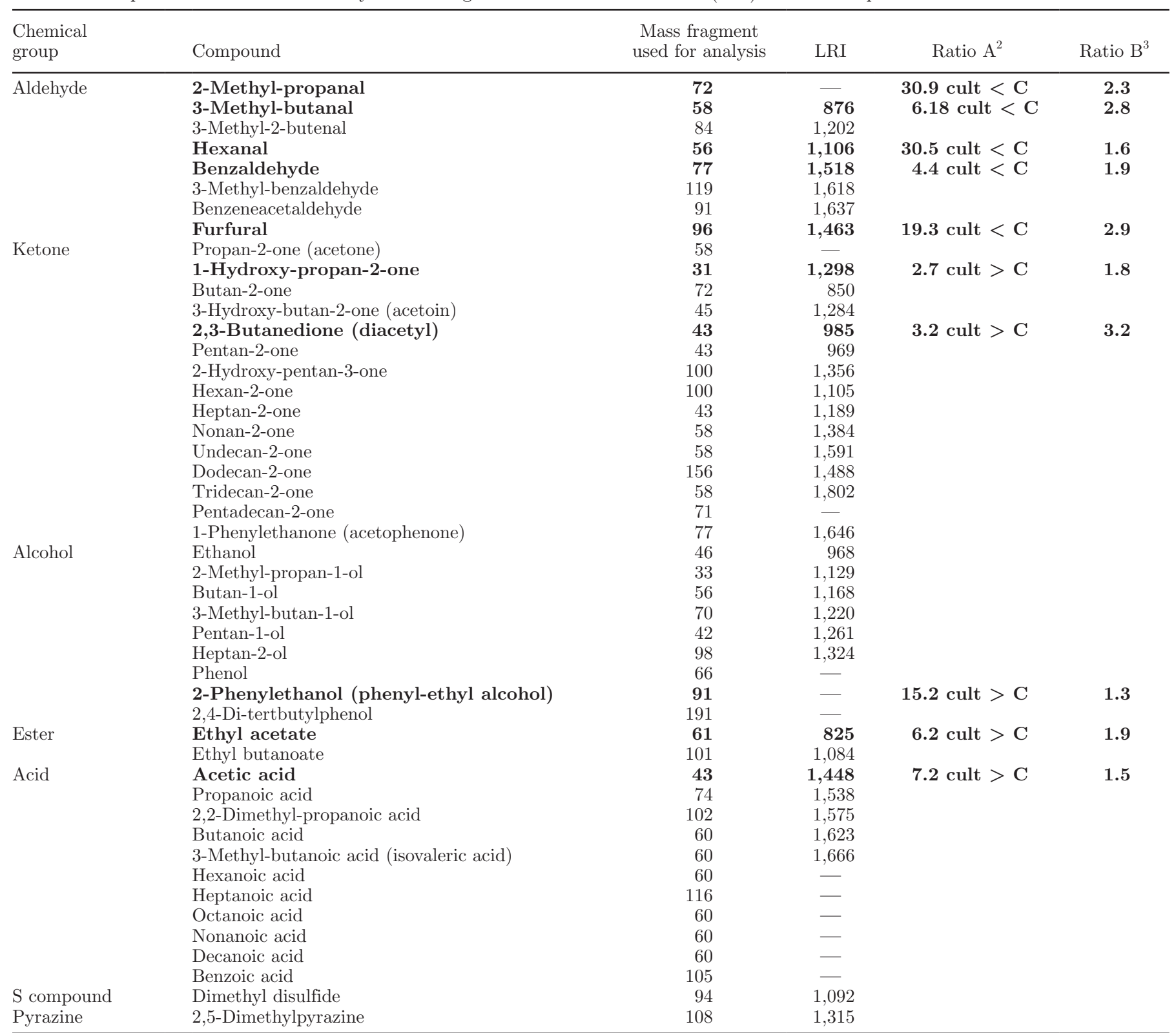

${ }^{1}$ The control was an uninoculated model under the same conditions. Cultures and control were tested in triplicate. Compounds that exhibited significant differences in abundances in cultures, including control $(P<0.05)$, are presented in bold.

${ }^{2}$ Ratio A presents the maximal ratio of abundance of a compound, between the cultures and the control: (abundance in cultures)/(abundance in control), if cult $>\mathrm{C}$; or (abundance in control)/(abundance in cultures), if cult $<\mathrm{C}$.

${ }^{3}$ Ratio B presents the maximal ratio of abundance of a compound between the cultures. 


\section{(a) Individuals factor map (PCA)}

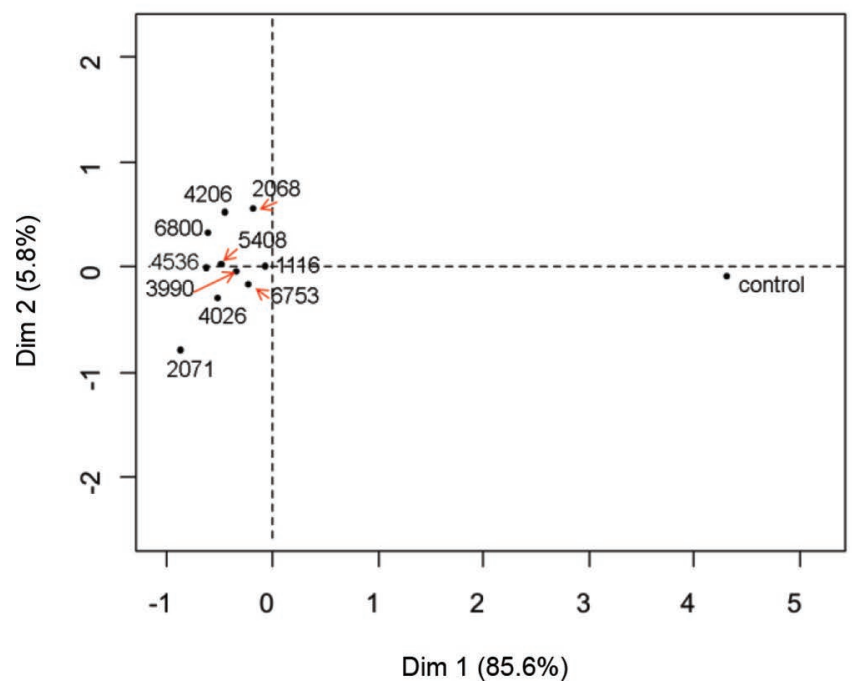

(b)

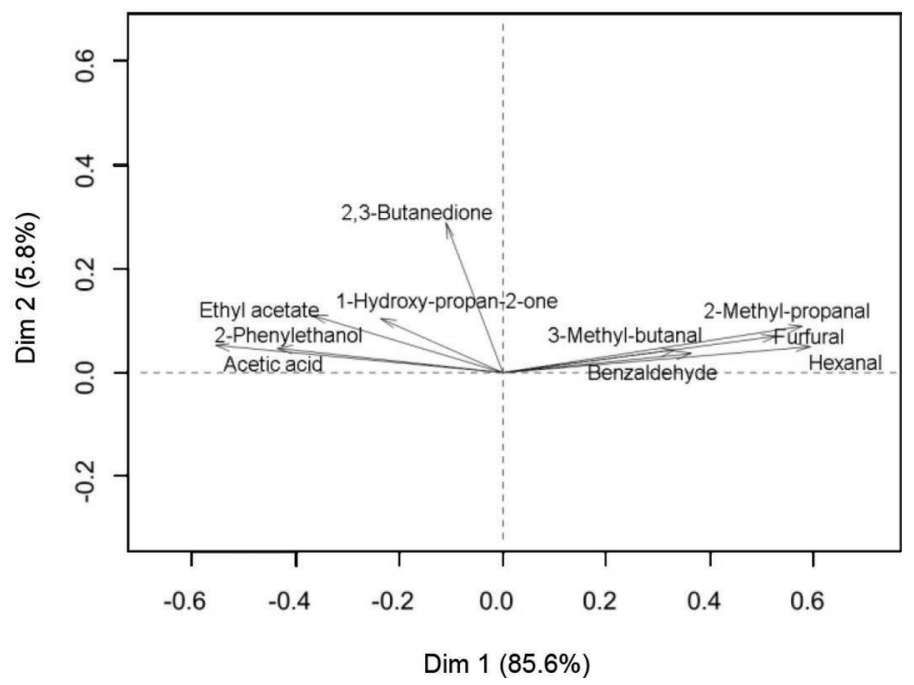

Figure 2. Individual factor map (a) and variable factor map (b) of principal component analysis (PCA) on 10 volatile compounds produced by 10 strains of Lactobacillus casei group in model system 2 incubated for $14 \mathrm{~d}$ at $30^{\circ} \mathrm{C}$. The control was an uninoculated model system incubated under the same conditions. Cultures and control were tested in triplicate. The DPC prefix has been removed from the strain name to avoid potential illegibility of the figure. Dim 1 = dimension 1; Dim $2=$ dimension 2. Color version available online.

nificant differences in relative abundances between the cultures was observed (Supplemental Table S2; https:/ / doi.org/10.3168/jds.2016-12408). The DPC4206 strain produced the highest abundance of 2,3-butanedione, DPC3990 produced the highest abundance of acetic acid, and DPC2068 produced the highest abundance of ethyl acetate.

In the PCA plot made for MS2 using the abundances of 10 volatiles across all cultures and the control (Figure 2), the first 2 axes accounted for $91 \%$ of the total variability. Dimension 1 described $85.6 \%$ of the variability. Five variables were negatively associated with PC1 (2,3-butanedione, 1-hydroxy-propan-2-one, ethyl acetate, acetic acid, and 2-phenylethanol), whereas all aldehydes were positively associated with PC1. Dimension 2, describing $5.8 \%$ of the variability, was mainly related to 2,3-butanedione. The control appeared in the right quadrant and was associated with the aldehydes. Dimension 1 was negatively associated with all the cultures. Conversely, $\mathrm{PC} 2$ was positively associated with DPC4206, DPC2068, and DPC6800, and negatively with DPC2071 and DPC4026. The compound that contributed the most to differentiation was 2,3-butanedione. The PCA showed that cultures were separated along PC2 according to the production of 2,3-butanedione, with DPC4206 and DPC2071 containing significantly the highest and the lowest amounts, respectively.

\section{DISCUSSION}

Strains of $L$. casei group represent one of the most frequently isolated NSLAB in cheese. Recently, it has been shown that NSLAB play the most pivotal role in flavor development of fermented dairy products, and it is considered that differences in their metabolic characteristics and activities could be a crucial factor for flavor diversification (Coolbear et al., 2008; Gobbetti et al., 2015). In this study, our objective was to analyze the metabolic biodiversity of 10 strains of the L. casei group, belonging to L. casei or L. paracasei species, by assessing their abilities to produce flavor-contributing compounds. To this end, 2 model systems were employed that differ in their constituents and incubation conditions. Model system 1 presents a highly concentrated mixture of $\mathrm{AA}$ at $\mathrm{pH}$ 7. In this model, our aim was to estimate the capability of strains to metabolize AA in optimal conditions for AA converting enzyme activity ( $\mathrm{pH}$ approximately 7 ). In MS2, we aimed to mimic the cheese environment providing different types of substrates (proteins, sugar, and lipids) and follow the metabolic activity of growing cells and cells in the stationary phase, as it occurs during cheese ripening.

Cell enumeration confirmed the expected behavior of strains in both model systems. In MS1, we did not observe any significant change in cell numbers, except for DPC2071, or changes in $\mathrm{pH}$ during incubation, except 
for 2 cultures, DPC2071 and DPC3990. As this model system contains a highly concentrated solution of AA, and very little of other nutrients that would support cell growth were present, cells did not grow. In contrast, MS2, provided the whole range of nutrients (sugar, proteins, and lipids), and cells numbers increased significantly $(P<0.05)$, whereas $\mathrm{pH}$ decreased, compared with the uninoculated control. Similar results were described in other studies where model systems were used. For example, in a synthetic model, Kieronczyk et al. (2001) showed only a slight decrease in the numbers of lactobacilli over $6 \mathrm{~d}$ of incubation. In cheese-based models, the number of $L$. plantarum increased over 30 d of incubation (Milesi et al., 2008), but a conflicting result was reported by Di Cagno et al. (2006) where numbers of mesophilic lactobacilli decreased by 1 log unit after $36 \mathrm{~d}$ of incubation. In the study of Pogačić et al. (2015), cell numbers of lactobacilli in a curdbased medium increased in the first $24 \mathrm{~h}$ of incubation, but after 5 wk the numbers of $L$. paracasei slightly decreased, reaching $8.14 \log _{10}$ units. The lower cell numbers reached in this study, using the same model system, could be due to the different incubation conditions (temperature and time).

In MS1, the aim was to determine the diversity of strains by their ability to metabolize AA, because the products of AA catabolism are generally seen as highly important food flavor contributors (Yvon and Rijnen, 2001). This model was developed on the basis of a model published by van de Bunt et al. (2014), which provided a rapid way to evaluate the flavor formation capacity of strains. In our approach, we used pancreatic digest of casein, because it brings AA in proportion similar to that of ripened cheese. In MS1, the inoculated strains produced volatiles such as shortchain aldehydes, alcohols, and acids that correspond to the intensive AA catabolism (McSweeney and Sousa, 2000). Butanal is known for its pungent flavor (Singh et al., 2003), whereas 3-methyl-butan-1-ol, a product of leucine metabolism, has a fruity, alcohol, grainy flavor (Singh et al., 2003). Aromatic alcohols, such as 2-phenylethanol arising from phenylalanine, have a rose flavor (Singh et al., 2003). Long-chain alcohols, such as undecan-2-ol, are produced in the reduction of 2-methyl ketones, and hexan-1-ol and octan-1-ol probably originated from the reduction of fatty acids. Long-chain ketones (undecan-2-one and tridecan-2-one) are products of lipid metabolism and have fruity, nutty notes (Collins et al., 2003). The aldehyde 2-ethyl-2-hexanal most probably originated during lipid oxidation. As inoculated cells were in the stationary phase of growth, some of fatty acids released from the cell membranes may have been the source of these compounds, as lipids were not present in this model system. Acids, such as butanoic and hexanoic acid, are characterized by rancid and goaty flavor, respectively (Curioni and Bosset, 2002), and have a lipid source, probably from the cell membranes, although hexanoic acid may also originate from lysine (Peralta et al., 2014). Branchedchain 3-methyl-butanoic acid, originated from leucine and has rancid, cheesy, and sweaty notes (Curioni and Bosset, 2002). Among the esters detected, butyl esters dominated, as a result of the secondary reaction of acid esterification due to the high abundance of butan-1-ol observed in this model system. This alcohol was present in the medium itself, since we detected it in the uninoculated control. Esters in general contribute to fruity flavor notes (Curioni and Bosset, 2002). Sulfur compounds that arise from sulfur AA (methionine, cysteine) metabolism contribute to garlic and onion flavors (Singh et al., 2003). Of all the sulfur compounds detected, the production of S-methyl-thioacetate showed the highest variations among cultures. This molecule is generated in the reaction of acetyl-CoA and methanethiol, a metabolite of methionine, and has a cooked cauliflower flavor (Arfi et al., 2002). The DMTS and dimethyl tetrasulfide were present in the highest concentration in the control. Their presence in the control may be a result of methionine degradation during the medium manufacture process, or its sterilization. Lower concentrations in the cultures compared with the control could also suggest that either DMTS and dimethyl tetrasulfide, or one of their precursors, such as methanethiol, may have been metabolized by the strains. In addition, whereas pyrazine derivatives were present in the control presumably originating due to the sterilization of the medium, significant differences between cultures were observed for 2 pyrazines (2,3,5-trimethyl-6-ethylpyrazine and 2,6-dimethyl3-sec-butylpyrazine) and it appears that the cultures may be producing these compounds, which contribute earthy, roasty, and potato flavors (Curioni and Bosset, 2002; Singh et al., 2003). Some bacteria and yeast can produce pyrazines (Schulz and Dickschat, 2007; Rajini et al., 2011), although an enzymatic pathway involved in pyrazine synthesis in lactobacilli has not been described. Pyrazines may arise in nonenzymatic reactions between metabolites of AA, such as $\alpha$-aminoketones and $\alpha$-dicarbonyl compounds (Rajini et al., 2011). A low level of total carbohydrates was present in the MS1, and as expected, we observed a limited number of sugar metabolites, including ethanol, acetic acid, which has typical vinegar flavor, and 3-hydroxy-butan-2-one, which is important for its buttery notes (Singh et al., 2003). However, both 3-hydroxy-butan-2-one and acetic acid could have also originated through AA metabolism (Skeie et al., 2008; Peralta et al., 2014). Nevertheless, we did not detect 2,3-butanedione, a compound 
produced from pyruvate, an intermediate molecule in carbohydrate metabolism (Jyoti et al., 2003; Liu, 2003; Bachmann et al., 2009). Additionally, in MS1, we identified 4-propylbenzaldehyde (most probably metabolite of phenylalanine) and 2-acetylthiazole [most probably originating from methionine, or cysteine (Law, 1997)]. These compounds are inevitably produced in the AArich environment, and although some of them have flavor potential (2-acetylthiazole; Burdock, 2016), they are not usually, if at all, associated with cheese flavor.

In MS1, strains showed considerable metabolic differences, with 3 strains, DPC2071, DPC3990, and DPC4206, producing the highest amounts of flavor compounds, often significantly higher compared with the production by other strains (LSD test, Supplemental Table S1; https://doi.org/10.3168/jds.2016-12408). This observation confirms the biodiversity of $L$. casei strains in their ability to metabolize AA and produce a variety of volatile compounds.

In MS2, we examined the biodiversity of strains not only in the presence of AA, but also in the presence of other substrates available in the processed curd or added during model preparation (lactose), to investigate their glycolytic and lipolytic activities. The main metabolic product of strains was 2,3-butanedione, which is considered as a major flavor contributor to buttery and cheesy notes (Curioni and Bosset, 2002) arising from lactose or citrate metabolism (Bachmann et al., 2009). Moreover, we observed the highest variability among tested cultures in the production of 2,3-butanedione, with DPC4206 and DPC2071 producing the highest and the lowest abundance, respectively. The aromatic alcohol 2-phenylethanol, a product of phenylalanine metabolism known for its rose flower notes (Curioni and Bosset, 2002), was also detected, with all the strains producing similar amounts of this alcohol. The strains also produced acetic acid from AA or carbohydrate sources (Singh et al., 2003), and ethyl acetate, which gives fruity notes. Esters originated from esterification of the acids and alcohols formed from carbohydrate and AA metabolism. Although many acids were detected in cultures, only 2 esters were identified. The reason for this observation could be the lower level of alcohols available or the dominance of the reverse reaction over the course of incubation time. The chromatograms were abundant in long-chain methyl-ketones and acids, but no significant difference was present between the abundances observed between the cultures and the control. These molecules most likely arose from lipid hydrolysis and the metabolism of starter cultures present in nonprocessed cheese curd (McSweeney and Sousa, 2000; Singh et al., 2003) and lipid oxidation probably occurred independently of the cultures metabolic activities, during sterilization, which contributed to the equal amounts of these compounds in all cultures and the control. All aldehydes, for which significant differences were observed, were present in lower concentrations in cultures than in the control, and probably were reduced to alcohols during incubation. The initial presence of aldehydes in the control could be connected to the metabolic activity of starter cultures present in the fresh curd.

Although cultures showed different abilities to metabolize substrates in MS2 and we observed differences in compound abundances in cultures compared with the control, the diversity of microbial volatiles among cultures was lower than observed using MS1, as only a few compounds were produced in significantly different abundances across the strains. 2,3-Butanedione was the molecule that contributed to the highest level of differentiation, as the ratio between the highest and the lowest abundance among the cultures was the highest for this compound. DPC4206 was shown to be the most differentiated from the other strains, producing the highest amount of 2,3-butanedione, followed by DPC6800 and DPC2068 (Supplemental Table S2; https://doi.org/10.3168/jds.2016-12408). Other compounds have also contributed to differentiation, but their effect was modest, as differences in abundances were lower. Although in PCA plot DPC2071 appears differentiated, its position was mainly due to a low level of aldehydes in comparison to the other cultures.

As an outcome of the diversity studies, a comparison of the 2 model systems was possible. First, in both model systems, we observed a difference between the control and the cultures, which suggests that all the strains were metabolically active in both environments. Model system 1 enabled detection of more flavor-related compounds compared with the MS2, but not all were directly associated with cheese flavor. Model system 1 is rich in AA, and as expected, this was the major pathway that could be investigated in this study with that model. Model system 1 provides a rapid approach for estimation of the ability of strains to metabolize $\mathrm{AA}$ in ratios present in final stages of cheese ripening. On the other hand, MS2, based on curd, enabled different flavor pathway development and also evaluated growing strains in conditions simulating cheese ripening $(\mathrm{NaCl}$ and te presence of other cheese substrates in corresponding amounts and ratios). This model allowed determination of volatiles produced by both growing cells (first $24-48 \mathrm{~h}$ of incubation) and cells in stationary phase (until the end of incubation). The profiles of MS1 were abundant in sulfur compounds that arose from methionine metabolism. However, although some of these are seen as flavor contributors, they are not often observed in cheese. Conversely, in MS2, only one sulfur compound (dimethyl disulfide) was detected. 
Compounds such as 2,3-butanedione and propionic acid originate from sugar and AA metabolism and were not present in MS1, but we identified them in MS2. Esterification was much more efficient in MS1 with butyl esters dominant, due to the extremely high abundance of butan-1-ol in the substrate. Conversely, only 2 ethyl esters were identified in MS2. However, in both model systems we confirmed that tested strains of the $L$. casei group have different abilities to metabolize substrates and produce a variety of compounds with potential to contribute to cheese flavor.

\section{CONCLUSIONS}

In this study, we aimed to investigate diversity of 10 strains of $L$. casei group based on their flavor-contributing potential. The metabolic variability of the strains was evaluated in 2 model systems. The results obtained in MS1 demonstrated that tested strains have different abilities to metabolize AA to flavor compounds, with strains DPC2071, DPC3990, and DPC4206 displaying the most diverse metabolic profiles. In MS2, strains used various metabolic pathways, and apart from volatiles produced through AA catabolism, metabolites originating from glycolysis and lipolysis were also identified, but differences between the strains were less evident and only strain DPC4206 was slightly different from the other strains. Taking all these results into account, we can conclude that strains of $L$. casei group express diverse metabolic potential in the 2 model systems. The use of model systems gave an insight into the metabolic characteristics and flavor development potential of the strains. The differences observed in volatile production can serve as guidance for selection of strains with the potential to diversify cheese flavor. It is envisaged that strain-to-strain diversity in volatile profiles will be reflected in variations in the flavor of manufactured cheese. The screening of volatile profiles of strains in model systems prior to cheese manufacture could help in selection of strains with potential to diversify cheese flavor.

\section{ACKNOWLEDGMENTS}

Ewelina Stefanovic and Andrea Bertuzzi are in a receipt of Teagasc Walsh Fellowships. This collaboration was supported by a Short Term Overseas Training Award 2015, funded by Teagasc.

\section{REFERENCES}

Arfi, K., H. E. Spinnler, R. Tache, and P. Bonnarme. 2002. Production of volatile compounds by cheese-ripening yeasts: Requirement for a methanethiol donor for S-methyl thioacetate synthesis by
Kluyveromyces lactis. Appl. Microbiol. Biotechnol. 58:503-510. https://doi.org/10.1007/s00253-001-0925-0.

Bachmann, H., Z. Kruijswijk, D. Molenaar, M. Kleerebezem, and J. E. van Hylckama Vlieg. 2009. A high-throughput cheese manufacturing model for effective cheese starter culture screening. J. Dairy Sci. 92:5868-5882. https://doi.org/10.3168/jds.2009-2372.

Banks, J. M., and A. G. Williams. 2004. The role of the nonstarter lactic acid bacteria in Cheddar cheese ripening. Int. J. Dairy Technol 57:145-152. https://doi.org/10.1111/j.1471-0307.2004.00150.x.

Bouton, Y., S. Buchin, G. Duboz, S. Pochet, and E. Beuvier. 2009. Effect of mesophilic lactobacilli and enterococci adjunct cultures on the final characteristics of a microfiltered milk Swiss-type cheese. Food Microbiol. 26:183-191. https://doi.org/10.1016/j. fm.2008.09.002.

Budinich, M. F., I. Perez-Diaz, H. Cai, S. A. Rankin, J. R. Broadbent, and J. L. Steele. 2011. Growth of Lactobacillus paracasei ATCC 334 in a cheese model system: A biochemical approach. J. Dairy Sci. 94:5263-5277. https://doi.org/10.3168/jds.2009-2512.

Burdock, G. A. 2016. Fenaroli's Handbook of Flavor Ingredients. 6th ed. CRC Press, Boca Raton. FL.

Cavanagh, D., K. N. Kilcawley, M. G. O'Sullivan, G. F. Fitzgerald, and O. McAuliffe. 2014. Assessment of wild non-dairy lactococcal strains for flavour diversification in a mini-Gouda type cheese model. Food Res. Int. 62:432-440. https://doi.org/10.1016/j foodres.2014.03.043.

Collins, Y. F., P. L. H. McSweeney, and M. G. Wilkinson. 2003. Lipolysis and free fatty acid catabolism in cheese: A review of current knowledge. Int. Dairy J. 13:841-866. https://doi.org/10.1016/ S0958-6946(03)00109-2.

Coolbear, T., V. Crow, J. Harnett, S. Harvey, R. Holland, and F. Martley. 2008. Developments in cheese microbiology in New Zealand-Use of starter and non-starter lactic acid bacteria and their enzymes in determining flavour. Int. Dairy J. 18:705-713. https:// doi.org/10.1016/j.idairyj.2008.03.010.

Crow, V., B. Curry, and M. Hayes. 2001. The ecology of non-starter lactic acid bacteria (NSLAB) and their use as adjuncts in New Zealand Cheddar. Int. Dairy J. 11:275-283. https://doi.org/10.1016/ S0958-6946(01)00057-7.

Curioni, P. M. G., and J. O. Bosset. 2002. Key odorants in various cheese types as determined by gas chromatography-olfactometry. Int. Dairy J. 12:959-984. https://doi.org/10.1016/S09586946(02)00124-3.

Di Cagno, R., M. Quinto, A. Corsetti, F. Minervini, and M. Gobbetti. 2006. Assessing the proteolytic and lipolytic activities of single strains of mesophilic lactobacilli as adjunct cultures using a Caciotta cheese model system. Int. Dairy J. 16:119-130. https://doi. $\operatorname{org} / 10.1016 /$ j.idairyj.2005.01.012.

Engels, W. J. M., and S. Visser. 1996. Development of cheese flavour from peptides and amino acids by cell-free extracts of Lactococcus lactis subsp cremoris B78 in a model system. Neth. Milk Dairy J. 50:3-17.

Fitzsimons, N. A., T. M. Cogan, S. Condon, and T. Beresford. 2001 Spatial and temporal distribution of non-starter lactic acid bacteria in Cheddar cheese. J. Appl. Microbiol. 90:600-608.

Gobbetti, M., M. De Angelis, R. Di Cagno, L. Mancini, and P. F. Fox. 2015. Pros and cons for using non-starter lactic acid bacteria (NSLAB) as secondary/adjunct starters for cheese ripening. Trends Food Sci. Technol. 45:167-178. https://doi.org/10.1016/j tifs.2015.07.016

Jyoti, B., A. Suresh, and K. Venkatesh. 2003. Diacetyl production and growth of Lactobacillus rhamnosus on multiple substrates. World J. Microbiol. Biotechnol. 19:509-514.

Kieronczyk, A., S. Skeie, K. Olsen, and T. Langsrud. 2001. Metabolism of amino acids by resting cells of non-starter lactobacilli in relation to flavour development in cheese. Int. Dairy J. 11:217-224. https://doi.org/10.1016/S0958-6946(01)00051-6.

Law, B. A. 1997. Microbiology and Biochemistry of Cheese and Fermented Milk. 2nd ed. Blackie Academic \& Professional, London, UK.

Liu, S. 2003. Practical implications of lactate and pyruvate metabolism by lactic acid bacteria in food and beverage fermentations. 
Int. J. Food Microbiol. 83:115-131. https://doi.org/10.1016/ s0168-1605(02)00366-5.

Marilley, L., and M. G. Casey. 2004. Flavours of cheese products: metabolic pathways, analytical tools and identification of producing strains. Int. J. Food Microbiol. 90:139-159. https://doi. org/10.1016/s0168-1605(03)00304-0.

McSweeney, P. L. H., and M. J. Sousa. 2000. Biochemical pathways for the production of flavour compounds in cheeses during ripening: A review. Lait 80:293-324.

Milesi, M. M., M. Candioti, and E. Hynes. 2007. Mini soft cheese as a simple model for biochemical studies on cheese-making and ripening. LWT Food Sci. Technol. (Campinas) 40:1427-1433. https:// doi.org/10.1016/j.lwt.2006.08.003.

Milesi, M. M., P. L. McSweeney, and E. R. Hynes. 2008. Viability and contribution to proteolysis of an adjunct culture of Lactobacillus plantarum in two model cheese systems: Cheddar cheese-type and soft-cheese type. J. Appl. Microbiol. 105:884-892. https://doi. org/10.1111/j.1365-2672.2008.03813.x.

Milesi, M. M., I. V. Wolf, C. V. Bergamini, and E. R. Hynes. 2010. Two strains of nonstarter lactobacilli increased the production of flavor compounds in soft cheeses. J. Dairy Sci. 93:5020-5031. https://doi.org/10.3168/jds.2009-3043.

Peralta, G. H., I. V. Wolf, C. V. Bergamini, M. C. Perotti, and E. R. Hynes. 2014. Evaluation of volatile compounds produced by Lactobacillus paracasei I90 in a hard-cooked cheese model using solid-phase microextraction. Dairy Sci. Technol. 94:73-81. https:// doi.org/10.1007/s13594-013-0143-4.

Pogačić, T., M. B. Maillard, A. Leclerc, C. Herve, V. Chuat, F. Valence, and A. Thierry. 2016. Lactobacillus and Leuconostoc volatilomes in cheese conditions. Appl. Microbiol. Biotechnol. 100:23352346. https://doi.org/10.1007/s00253-015-7227-4.

Pogačić, T., M. B. Maillard, A. Leclerc, C. Herve, V. Chuat, A. L. Yee, F. Valence, and A. Thierry. 2015. A methodological approach to screen diverse cheese-related bacteria for their ability to produce aroma compounds. Food Microbiol. 46:145-153. https://doi. org/10.1016/j.fm.2014.07.018

Rajini, K. S., P. Aparna, C. Sasikala, and V. Ramana Ch. 2011. Microbial metabolism of pyrazines. Crit. Rev. Microbiol. 37:99-112. https://doi.org/10.3109/1040841X.2010.512267.

Randazzo, C. L., S. De Luca, A. Todaro, C. Restuccia, C. M. Lanza, G. Spagna, and C. Caggia. 2007. Preliminary characterization of wild lactic acid bacteria and their abilities to produce flavour compounds in ripened model cheese system. J. Appl. Microbiol. 103:427-435. https://doi.org/10.1111/j.1365-2672.2006.03261.x.

Schulz, S., and J. S. Dickschat. 2007. Bacterial volatiles: The smell of small organisms. Nat. Prod. Rep. 24:814-842. https://doi. org/10.1039/b507392h.

Sgarbi, E., C. Lazzi, G. Tabanelli, M. Gatti, E. Neviani, and F. Gardini. 2013. Nonstarter lactic acid bacteria volatilomes produced using cheese components. J. Dairy Sci. 96:4223-4234. https://doi. org/10.3168/jds.2012-6472.

Singh, S., and R. Singh. 2014. Phenotypic and genotypic characterization of non-starter Lactobacillus species diversity in Indian Cheddar cheese. LWT Food Sci. Technol. (Campinas) 55:415-420. https://doi.org/10.1016/j.lwt.2013.09.018.

Singh, T., M. Drake, and K. Cadwallader. 2003. Flavor of Cheddar cheese: A chemical and sensory perspective. Compr. Rev. Food Sci. Food Saf. 2:166-189

Skeie, S., A. Kieronczyk, R. M. Næss, and H. Østlie. 2008. Lactobacillus adjuncts in cheese: Their influence on the degradation of citrate and serine during ripening of a washed curd cheese. Int. Dairy J. 18:158-168. https://doi.org/10.1016/j.idairyj.2007.09.003.

Smit, G., A. Braber, W. vanSpronsen, G. VandenBerg, and F. A. Exterkate. 1995. Ch-easy model: A cheese-based model to study cheese ripening. Bioflavour 95:185-190.

Smit, G., B. A. Smit, and W. J. Engels. 2005. Flavour formation by lactic acid bacteria and biochemical flavour profiling of cheese products. FEMS Microbiol. Rev. 29:591-610. https://doi. org/10.1016/j.femsre.2005.04.002.

Smith, C. A., E. J. Want, G. O'Maille, R. Abagyan, and G. Siuzdak. 2006. XCMS: Processing mass spectrometry data for metabolite profiling using nonlinear peak alignment, matching, and identification. Anal. Chem. 78:779-787. https://doi.org/10.1021/ac051437y.

Stefanovic, E., K. N. Kilcawley, M. C. Rea, G. F. Fitzgerald, and O. McAuliffe. 2017. Genetic, enzymatic and metabolite profiling of the Lactobacillus casei group reveals strain biodiversity and potential applications for flavour diversification. J. Appl. Microbiol. https://doi.org/10.1111/jam.13420.

Swearingen, P. A., D. J. O'Sullivan, and J. J. Warthesen. 2001. Isolation, characterization, and influence of native, nonstarter lactic acid bacteria on Cheddar cheese quality. J. Dairy Sci. 84:50-59. https://doi.org/10.3168/jds.S0022-0302(01)74451-7.

van de Bunt, B., P. A. Bron, L. Sijtsma, W. M. de Vos, and J. Hugenholtz. 2014. Use of non-growing Lactococcus lactis cell suspensions for production of volatile metabolites with direct relevance for flavour formation during dairy fermentations. Microb. Cell Fact. 13:176.

Vaughan, C., B. Curry, and M. Hayes. 2001. The ecology of nonstarter lactic acid bacteria (NSLAB) and their use as adjuncts in New Zealand Cheddar. Int. Dairy J. 11:215-283.

Velez, M. A., M. C. Perotti, S. R. Rebechi, and E. R. Hynes. 2015. Short communication: A new minicurd model system for hard cooked cheeses. J. Dairy Sci. 98:3679-3683. https://doi.org/10.3168/ jds.2014-8906.

Yvon, M., and L. Rijnen. 2001. Cheese flavour formation by amino acid catabolism. Int. Dairy J. 11:185-201. https://doi.org/10.1016/ S0958-6946(01)00049-8. 